\title{
Immunohistochemical evaluation of Ki-67, PCNA and MCM2 proteins proliferation index (PI) in advanced gastric cancer
}

\author{
Jolanta Czyżewska1, Katarzyna Guzińska-Ustymowicz², Anna Pryczynicz², \\ Andrzej Kemona ${ }^{2}$, Roman Bandurski ${ }^{3}$
}

\author{
${ }^{1}$ Department of Clinical Laboratory Diagnostics, ${ }^{2}$ Department of General Pathomorphology and \\ ${ }^{3}$ Department of General and Gastroenterological Surgery \\ Medical University of Bialystok, Bialystok, Poland.
}

\begin{abstract}
The current study objective was to assess the proliferation indices (PI) of Ki-67, PCNA and MCM2 proteins in advanced gastric cancer and in metastatic lymph node in correlation with certain clinicopathological features and with postoperative survival of patients. The study was conducted in a group of 100 patients with advanced gastric cancers. Involvement of local lymph nodes was present in 36 cases. Immunohistochemical investigations were carried out using monoclonal antibodies against Ki-67 (DAKO), PCNA (DAKO) and polyclonal antibody to MCM2 (Santa Cruz Biotechnology). Visualization of the antigen/antibody complex was performed using LSAB technique (biotin-streptavidin-peroxidase) followed by application of chromogene DAB (DAKO). Statistical analysis revealed no correlations of Ki-67, PCNA and MCM2 PI in tumour tissue or metastatic lymph node with patients' age and gender, tumour location, histological grade, macroscopic type according to Bormann's classification and histological grading by Lauren's and Goseki's classifications. Moreover, no correlation was observed of Ki-67 and MCM2 PI in tumour tissue with histological grading. No correlation was also noted between the proliferation indices of all the three proteins in the affected lymph node and grade of histological differentiation. Such clinicopathological parameters as patients' age and gender, histological grading by Lauren's and Goseki's classifications and lymph node involvement did not correlate with survival time of patients. Furthermore, no statistically significant correlation was shown of postoperative survival time with Ki-67 and MCM2 PI in tumour tissue and metastatic lymph nodes and with PCNA PI in the affected lymph nodes. However, a statistically significant correlation was found of Ki-67, PCNA and MCM2 PI in tumour tissue and metastatic lymph nodes with depth of wall invasion and local lymph node involvement. A statistically significant correlation was also noted between PCNA PI in the main mass of tumour and histological grading. The postoperative survival time of patients exhibited a statistically significant correlation with tumour location and macroscopic type according to Bormann's classification. Correlations on statistical borderline were noted between survival time and depth of gastric wall invasion and PCNA PI in the main mass of tumour.
\end{abstract}

Key words: Ki-67, PCNA, MCM2, proliferation index, gastric carcinoma.

\section{Introduction}

Gastric carcinoma is one of the most common malignancies in the world. In the second half of the $20^{\text {th }}$ century, morbidity and mortality rates due to gastric cancer showed a sudden increase [1]. Tumour growth is

Correspondence: J. Czyżewska, Dept. of Clinical Laboratory Diagnostics, Medical University of Białystok, Waszyngtona 15a 15-274 Białystok, Poland; tel.: (+4885) 7485942, fax.: (+4885) 7485990, e-mail: czyzyk15@op.pl characterized by dominance of cell proliferation over cell death, accompanied by a simultaneous suppression of differentiation. This process occurs uncontrollably and is harmful to the body, resulting in the formation of incompletely differentiated tissue with a tendency towards continuous extension. Cancer proliferation is caused by irreversible changes in the cell genome, leading to dissociation of regulatory and repair mechanisms. As revealed by recent literature reports, PI determination may provide prognostically important information in various types of cancers. 
The most widely used proliferation markers include $\mathrm{Ki}-67, \mathrm{PCNA}$ (proliferation cell nuclear antigen) and MCM2 (minichromosome maintenance protein 2). The Ki-67 protein was discovered by Gerdes in 1983 as a prototype monoclonal antibody $\mathrm{Ki}-67 . \mathrm{Ki}-67$ interacts with the nuclear structure present in the nucleus only in actively proliferating cells, i.e. during the G1, S and G2 phases of the cell cycle and mitosis. Non-dividing or "resting" cells in the G0 phase are Ki67 antigen negative [2-4]. PCNA is a 36-kDa auxiliary protein for DNA polymerase $\delta$ and $\varepsilon$ [5]. The PCNA protein plays a major role in metabolism of nucleic acids and has been found to interact with a number of proteins involved in DNA replication and repair, thus protecting the DNA sequence pattern [6]. It is synthesized in the cell nucleus and has a key role in cell cycle initiation. The concentration of PCNA correlates with cell proliferation - it shows a rise during the G1 phase, reaches the peak during the G1, S and interphase, falls during the $\mathrm{G} 2$, reaches the low in the M phase, and is untraceable in cells that are out of the cell cycle [7]. The minichromosome maintenance protein family (MCM) includes at least six various nuclear proteins (MCM2-7), showing a striking sequential homology of 200 amino acids in the central region [8]. MCM proteins are essential for DNA replication as part of a prereplication complex (pre-RC). The activation of the MCM complex, through cyclin-dependent kinases and the Cdc7 (SpHsk1) protein kinase, initiates DNA synthesis [9]. Moreover, the MCM complex has been found to interact with histone $\mathrm{H} 3$ that stabilizes interactions between MCM proteins and chromatin or affects nucleosome stability in the vicinity of replication forks [10]. MCM2 protein is absent in cells that have left the cycle (G0), but is present in proliferating cells [11]. The study objective was to assess the proliferation indices of Ki-67, PCNA and MCM2 proteins in advanced gastric cancer and metastatic lymph node in correlation with certain clinicopathological features and survival of patients.

\section{Material and methods}

Collection of samples. The study group consisted of 100 chosen patients with advanced gastric carcinoma (33 women and $67 \mathrm{men}$ ), operated on in the years 1989-2003. The mean age was 62.5 years (range 27-83). The patients were clinically monitored for 84 months.

Immunohistochemistry. Formalin-fixed and paraffin-embedded tissue specimens were cut on a microtome into $5 \mu \mathrm{m}$ thick sections. The sections were deparaffinised in xylenes and hydrated in alcohols of decreasing concentration. In order to visualise the antigen, the sections were heated in citrate buffer $\mathrm{pH} 6.0$ (in a pressure cooker for $3 \mathrm{~min}$. for Ki-67 and PCNA and in a microwave oven, $750 \mathrm{~W}$, for $15 \mathrm{~min}$. for MCM2). After cooling to the room temperature, in order to block endogenous peroxidase activity, 10-min incubation was performed with $\mathrm{H}_{2} \mathrm{O}_{2}$. MCM2-positive sections were incubated with $\mathrm{H}_{2} \mathrm{O}_{2}$ and methanol for $15 \mathrm{~min}$., and then with normal blocking serum (goat ABC Staining System, cat. no sc2023, Santa Cruz Biotechnology). After rinsing in TRIS buffer, $\mathrm{pH}=7.4$, for $10 \mathrm{~min}$, the sections were incubated with antibodies: Ki-67 (mouse monoclonal antibody, clone MIB-1, cat. no. M7240, DAKO) in a dilution of 1:100 for 60 min; PCNA (mouse monoclonal antibody, clone PC10, cat. no. M0879, DAKO) in a dilution of 1:400 for $60 \mathrm{~min}$ and MCM2 (goat polyclonal antibody, cat. no sc-9839, Santa Cruz Biotechnology) in a dilution of 1:300 overnight at a room temperature. In the next stage, biotinyl antibody was used in the reaction and then the streptavidin-peroxidase complex (LASB ${ }^{\circledR}+$ System- HRP, nr kat. K0690, DAKO) was applied. The antigen-antibody complex was visualized by DAB chromogen (3'3- diamonobenzidine, cat. no. S3000, DAKO).

Sample assessment. The reactions for Ki-67, PCNA and MCM2 proteins were observed in the cell nucleus. Protein expression was assessed using a light microscope at a magnification of $60 \times$. The percentage of protein-positive cells was determined semiquantitatively for each 10 fields of vision, as compared to all the cells visible there. The mean from all the fields of vision was then calculated, giving the proliferation index expressed in $\%$ for each protein.

Next, based on the index, two study groups were isolated in which nuclear accumulation of Ki-67, PCNA and MCM2 in cancer cells was determined as either

- negative (-) - lack of reaction for Ki-67, PCNA and MCM2 or reaction present in $<50 \%$ of cancer cells, or

- positive $(+)$ - reaction for Ki-67, PCNA and MCM2 present $/$ visible in $>50 \%$ of cancer cells.

Statistical analysis. Statistical analysis was based on test $\chi^{2}$ and exact Fisher's test. The $\mathrm{p}<0.05$ was considered statistically significant. Multivariate Cox regression analysis was done to evaluate the 5 -year overall survival.

\section{Results}

Statistical analysis did not show any correlations of Ki-67, PCNA and MCM2 PI in the main mass of tumour and metastatic lymph node with age and gender of patients or tumour location in the stomach. Moreover, no relationship was noted of the protein PI in tumour tissue and metastatic lymph node with macroscopic type in Bormann's classification and with histological type by Lauren's and Goseki's classifications. Furthermore, the proliferation indices of Ki-67 and MCM2 in tumour tissue and metastatic lymph node were not found to correlate with the grade of histological differentiation (Tables 1,2). No correlation was also found between PCNA PI in metastatic lymph node and histological grading. A statistically significant difference was observed of Ki-67, PCNA and MCM2 PI in the main mass of tumour with depth of invasion in the gastric wall $(\mathrm{p}=0.005 ; \mathrm{p}=0.0005$; $\mathrm{p}=0.04$ ) (Table 1) and lymph node involvement $(p=0.0000 ; p=0.000 ; p=0.000)$ (Table 1). The proliferation index of PCNA in tumour sections was found to correlate with histological grading $(\mathrm{p}=0.05)$ (Table 1). Similarly, a statistically significant relationship was noted of Ki-67, PCNA and MCM2 PI in metastatic lymph nodes with depth of wall invasion $(p=0.0 .009$; $\mathrm{p}=0.00001 ; \mathrm{p}=0.06)$ (Table 2$)$ and lymph node involvement $(\mathrm{p}=0.0 .0005 ; \mathrm{p}=0.0000 ; \mathrm{p}=0.007)$ (Table 2). 
Table 1. Correlation between chosen clinico-pathological parameters and proliferation index (PI) Ki-67, PCNA and MCM2 proteins in gastric carcinoma.

\begin{tabular}{|c|c|c|c|c|c|c|c|c|c|c|}
\hline \multirow{2}{*}{\multicolumn{2}{|c|}{ Parameters }} & \multicolumn{3}{|c|}{$\mathrm{Ki}-67$} & \multicolumn{3}{|c|}{ PCNA } & \multicolumn{3}{|c|}{ MCM2 } \\
\hline & & \multirow{2}{*}{$\begin{array}{c}\mathrm{PI} \\
<=50 \% \\
22 \\
(64,7 \%)\end{array}$} & \multirow{2}{*}{$\begin{array}{c}\begin{array}{c}\mathrm{PI}> \\
50 \%\end{array} \\
12 \\
(35,3 \%)\end{array}$} & \multirow{3}{*}{$\begin{array}{c}\mathrm{p} \\
\text { "NS }\end{array}$} & \multirow{2}{*}{$\begin{array}{c}\mathrm{PI} \\
<=50 \% \\
22 \\
(64,7 \%)\end{array}$} & \multirow{2}{*}{$\begin{array}{c}\mathrm{PI}> \\
50 \% \\
12 \\
(35,3 \%)\end{array}$} & \multirow{3}{*}{$\begin{array}{c}\mathrm{p} \\
\text { "NS }\end{array}$} & \multirow{2}{*}{$\begin{array}{c}\mathrm{PI} \\
<=50 \% \\
19 \\
(55,9 \%)\end{array}$} & \multirow{2}{*}{$\begin{array}{c}\mathrm{PI}> \\
50 \%\end{array}$} & \multirow{3}{*}{$\begin{array}{c}\mathrm{p} \\
\text { "NS }\end{array}$} \\
\hline$\Lambda g e$ & $<=60$ & & & & & & & & & \\
\hline & $>60$ & $\begin{array}{c}40 \\
(61,5 \%)\end{array}$ & $\begin{array}{c}25 \\
(38,5 \%)\end{array}$ & & $\begin{array}{c}38 \\
(58,5 \%)\end{array}$ & $\begin{array}{c}27 \\
(35 \%)\end{array}$ & & $\begin{array}{c}39 \\
(60 \%)\end{array}$ & $\begin{array}{c}26 \\
(40 \%)\end{array}$ & \\
\hline \multirow[t]{2}{*}{ Sex } & Male & $\begin{array}{c}43 \\
(64,2 \%)\end{array}$ & $\begin{array}{c}24 \\
(35,8 \%)\end{array}$ & \multirow[t]{2}{*}{ *NS } & $\begin{array}{c}44 \\
(65,7 \%)\end{array}$ & $\begin{array}{c}23 \\
(34,3 \%)\end{array}$ & \multirow[t]{2}{*}{ *NS } & $\begin{array}{c}40 \\
(59,7 \%)\end{array}$ & $\begin{array}{c}27 \\
(40,3 \%)\end{array}$ & \multirow[t]{2}{*}{ *NS } \\
\hline & Female & $\begin{array}{c}20 \\
(60,6 \%)\end{array}$ & $\begin{array}{c}13 \\
(39,4 \%)\end{array}$ & & $\begin{array}{c}17 \\
(51,5 \%)\end{array}$ & $\begin{array}{c}16 \\
(48,5 \%)\end{array}$ & & $\begin{array}{c}18 \\
(54,5 \%)\end{array}$ & $\begin{array}{c}15 \\
(45,5 \%)\end{array}$ & \\
\hline \multirow{3}{*}{$\begin{array}{l}\text { Tumor } \\
\text { localization } \\
\text { (stomach) }\end{array}$} & $\begin{array}{c}1 / 3 \text { of up } \\
\text { part }\end{array}$ & $\begin{array}{c}5 \\
(62,5 \%)\end{array}$ & $\begin{array}{c}3 \\
(37,5 \%)\end{array}$ & \multirow[t]{3}{*}{ *NS } & $\begin{array}{c}5 \\
(62,5 \%)\end{array}$ & $\begin{array}{c}3 \\
(37,5 \%)\end{array}$ & \multirow[t]{3}{*}{ *NS } & $\begin{array}{c}4 \\
(50 \%)\end{array}$ & $\begin{array}{c}4 \\
(50 \%)\end{array}$ & \multirow[t]{3}{*}{ *NS } \\
\hline & $\begin{array}{c}1 / 3 \text { of } \\
\text { middle part }\end{array}$ & $\begin{array}{c}22 \\
(56,4 \%)\end{array}$ & $\begin{array}{c}17 \\
(43,6 \%)\end{array}$ & & $\begin{array}{c}24 \\
(61,5 \%)\end{array}$ & $\begin{array}{c}15 \\
(38,5 \%)\end{array}$ & & $\begin{array}{c}22 \\
(57,9 \%)\end{array}$ & $\begin{array}{c}16 \\
(42,1 \%)\end{array}$ & \\
\hline & $\begin{array}{c}1 / 3 \text { of } \\
\text { down part }\end{array}$ & $\begin{array}{c}36 \\
(67,9 \%)\end{array}$ & $\begin{array}{c}17 \\
(32,1 \%)\end{array}$ & & $\begin{array}{c}32 \\
(60,4 \%)\end{array}$ & $\begin{array}{c}21 \\
(39,6 \%)\end{array}$ & & $\begin{array}{c}32 \\
(60,4 \%)\end{array}$ & $\begin{array}{c}21 \\
(39,6 \%)\end{array}$ & \\
\hline \multirow[t]{3}{*}{$\begin{array}{l}\text { Depth of } \\
\text { invasion }\end{array}$} & pT1 & $\begin{array}{c}11 \\
(91,7 \%)\end{array}$ & $\begin{array}{c}1 \\
(8,3)\end{array}$ & \multirow[t]{3}{*}{0.005} & $\begin{array}{c}11 \\
(91,7 \%)\end{array}$ & $\begin{array}{c}1 \\
(8,3 \%)\end{array}$ & \multirow[t]{3}{*}{0.0005} & $\begin{array}{c}11 \\
(91,7 \%)\end{array}$ & $\begin{array}{c}1 \\
(8,3 \%)\end{array}$ & \multirow[t]{3}{*}{0.04} \\
\hline & p12 & $\begin{array}{c}15 \\
(83,3 \%)\end{array}$ & $\begin{array}{c}3 \\
(16,7 \%)\end{array}$ & & $\begin{array}{c}16 \\
(88,9 \%)\end{array}$ & $\begin{array}{c}2 \\
(11,1 \%)\end{array}$ & & $\begin{array}{c}12 \\
(66,7 \%)\end{array}$ & $\begin{array}{c}6 \\
(33,3 \%)\end{array}$ & \\
\hline & $\mathrm{pT} 3 / 4$ & $\begin{array}{c}37 \\
(52,9 \%)\end{array}$ & $\begin{array}{c}1 \\
(8,3 \%)\end{array}$ & & $\begin{array}{c}34 \\
(48,8 \%)\end{array}$ & $\begin{array}{c}36 \\
(51,2 \%)\end{array}$ & & $\begin{array}{c}36 \\
(51,4 \%)\end{array}$ & $\begin{array}{c}34 \\
(48,6 \%)\end{array}$ & \\
\hline \multirow[t]{3}{*}{$\mathrm{pN}$} & No & $0(0 \%)$ & $0 \quad(0 \%)$ & \multirow[t]{3}{*}{0.0000} & $0 \quad(0 \%)$ & $0 \quad(0 \%)$ & \multirow[t]{3}{*}{0.0000} & $0 \quad(0 \%)$ & $0 \quad(0 \%)$ & \multirow[t]{3}{*}{0.0000} \\
\hline & N1 & $\begin{array}{c}7 \\
(43,8 \%)\end{array}$ & $\begin{array}{c}11 \\
(56,2 \%)\end{array}$ & & $\begin{array}{c}5 \\
(27,8 \%)\end{array}$ & $\begin{array}{c}13 \\
(72,2 \%)\end{array}$ & & $\begin{array}{c}10 \\
(55,6 \%)\end{array}$ & $\begin{array}{c}8 \\
(44,4 \%) \\
\end{array}$ & \\
\hline & N2 & $\begin{array}{c}3 \\
(16,7 \%)\end{array}$ & $\begin{array}{c}15 \\
(83,3 \%)\end{array}$ & & $\begin{array}{c}0 \\
(0 \%)\end{array}$ & $\begin{array}{c}18 \\
(100 \%)\end{array}$ & & $\begin{array}{c}4 \\
(22,2 \%)\end{array}$ & $\begin{array}{c}14 \\
(77,8 \%)\end{array}$ & \\
\hline \multirow[t]{4}{*}{$\begin{array}{l}\text { Bormann's } \\
\text { classification }\end{array}$} & I & $\begin{array}{c}5 \\
(71,4 \%)\end{array}$ & $\begin{array}{c}3 \\
(16,7 \%)\end{array}$ & ${ }^{*} \mathrm{NS}$ & $\begin{array}{c}6 \\
(85,7 \%)\end{array}$ & $\begin{array}{c}1 \\
(14,3 \%)\end{array}$ & *NS & $\begin{array}{c}4 \\
(57,1 \%)\end{array}$ & $\begin{array}{c}3 \\
(42,9 \%)\end{array}$ & *NS \\
\hline & II & $\begin{array}{c}14 \\
(70 \%)\end{array}$ & $\begin{array}{c}6 \\
(30 \%)\end{array}$ & & $\begin{array}{c}13 \\
(65 \%)\end{array}$ & $\begin{array}{c}7 \\
(35 \%)\end{array}$ & & $\begin{array}{c}14 \\
(70 \%)\end{array}$ & $\begin{array}{c}6 \\
(30 \%)\end{array}$ & \\
\hline & III & $\begin{array}{c}36 \\
(65,5 \%)\end{array}$ & $\begin{array}{c}19 \\
(34,5 \%)\end{array}$ & & $\begin{array}{c}35 \\
(63,6 \%)\end{array}$ & $\begin{array}{c}20 \\
(36,4 \%)\end{array}$ & & $\begin{array}{c}34 \\
(61,8 \%)\end{array}$ & $\begin{array}{c}21 \\
(38,2 \%)\end{array}$ & \\
\hline & IV & $\begin{array}{c}8 \\
(44,4 \%)\end{array}$ & $\begin{array}{c}10 \\
(55,6 \%)\end{array}$ & & $\begin{array}{c}7 \\
(38,9 \%)\end{array}$ & $\begin{array}{c}11 \\
(61,1 \%)\end{array}$ & & $\begin{array}{c}7 \\
(38,9 \%)\end{array}$ & $\begin{array}{c}11 \\
(61,1 \%)\end{array}$ & \\
\hline $\begin{array}{l}\text { Lauren's } \\
\text { classification }\end{array}$ & $\begin{array}{l}\text { Intestinal } \\
\text { type }\end{array}$ & $\begin{array}{c}45 \\
(64,3 \%)\end{array}$ & $\begin{array}{c}25 \\
(35,7 \%)\end{array}$ & *NS & $\begin{array}{c}44 \\
(62,9 \%)\end{array}$ & $\begin{array}{c}26 \\
(37,1 \%)\end{array}$ & ${ }^{*} \mathrm{NS}$ & $\begin{array}{c}40 \\
(57,1 \%)\end{array}$ & $\begin{array}{c}30 \\
(42,9 \%)\end{array}$ & ${ }^{*} \mathrm{NS}$ \\
\hline & $\begin{array}{l}\text { Diffuse } \\
\text { type }\end{array}$ & $\begin{array}{c}18 \\
(60 \%)\end{array}$ & $\begin{array}{c}12 \\
(40 \%)\end{array}$ & & $\begin{array}{c}17 \\
(56,7 \%)\end{array}$ & $\begin{array}{c}13 \\
(43,3 \%)\end{array}$ & & $\begin{array}{c}19 \\
(63,3 \%)\end{array}$ & $\begin{array}{c}11 \\
(36,7 \%)\end{array}$ & \\
\hline $\begin{array}{l}\text { Goseki's } \\
\text { classification }\end{array}$ & I & $\begin{array}{c}6 \\
(66,7 \%)\end{array}$ & $\begin{array}{c}3 \\
(33,3 \%)\end{array}$ & *NS & $\begin{array}{c}2 \\
(66,7 \%)\end{array}$ & $\begin{array}{c}1 \\
(33,3 \%)\end{array}$ & *NS & $\begin{array}{c}5 \\
(55,6 \%)\end{array}$ & $\begin{array}{c}4 \\
(44,4 \%)\end{array}$ & *NS \\
\hline & II & $\begin{array}{c}23 \\
(74,2 \%)\end{array}$ & $\begin{array}{c}8 \\
(25,8 \%)\end{array}$ & & $\begin{array}{c}3 \\
(33,3 \%)\end{array}$ & $\begin{array}{c}6 \\
(66,7 \%)\end{array}$ & & $\begin{array}{c}21 \\
(67,7 \%)\end{array}$ & $\begin{array}{c}10 \\
(32,3 \%)\end{array}$ & \\
\hline & III & $\begin{array}{c}11 \\
(61,1 \%)\end{array}$ & $\begin{array}{c}7 \\
(38,9 \%)\end{array}$ & & $\begin{array}{c}2 \\
(25 \%)\end{array}$ & $\begin{array}{c}6 \\
(75 \%)\end{array}$ & & $\begin{array}{c}10 \\
(55,6 \%)\end{array}$ & $\begin{array}{c}8 \\
(44,4 \%) \\
\end{array}$ & \\
\hline & IV & $\begin{array}{c}23 \\
(54,8 \%)\end{array}$ & $\begin{array}{c}19 \\
(45,2 \%)\end{array}$ & & $\begin{array}{c}3 \\
(18,8 \%)\end{array}$ & $\begin{array}{c}13 \\
(81,2 \%)\end{array}$ & & $\begin{array}{c}23 \\
(54,8 \%)\end{array}$ & $\begin{array}{c}19 \\
(45,2 \%)\end{array}$ & \\
\hline $\begin{array}{l}\text { Histological } \\
\text { differentiation }\end{array}$ & G2 & $\begin{array}{c}32 \\
(69,6 \%)\end{array}$ & $\begin{array}{c}14 \\
(30,4 \%)\end{array}$ & ${ }^{*} \mathrm{NS}$ & $\begin{array}{c}32 \\
(69,6 \%)\end{array}$ & $\begin{array}{c}14 \\
(30,4 \%)\end{array}$ & 0.05 & $\begin{array}{c}29 \\
(64,4 \%)\end{array}$ & $\begin{array}{c}16 \\
(35,6 \%)\end{array}$ & ${ }^{*} \mathrm{NS}$ \\
\hline & G3 & $\begin{array}{c}30 \\
(55,6 \%)\end{array}$ & $\begin{array}{c}24 \\
(44,4 \%)\end{array}$ & & $\begin{array}{c}28 \\
(51,9 \%)\end{array}$ & $\begin{array}{c}26 \\
(48,1 \%)\end{array}$ & & $\begin{array}{c}29 \\
(53,7 \%)\end{array}$ & $\begin{array}{c}25 \\
(46,3 \%)\end{array}$ & \\
\hline
\end{tabular}

*NS - non statistical significence 
Table 2. Correlation between chosen clinico-pathological parameters and proliferation index (PI) Ki-67, PCNA and MCM2 proteins in lymph node metastasis

\begin{tabular}{|c|c|c|c|c|c|c|c|c|c|c|}
\hline \multirow{2}{*}{\multicolumn{2}{|c|}{ Parameters }} & \multicolumn{3}{|c|}{ Ki-67 } & \multicolumn{3}{|c|}{ PCNA } & \multicolumn{3}{|c|}{ MCM2 } \\
\hline & & \multirow{2}{*}{$\begin{array}{c}\mathrm{PI} \\
<=50 \% \\
3 \\
(25 \%)\end{array}$} & \multirow{2}{*}{$\begin{array}{c}\mathrm{PI}> \\
50 \% \\
9 \\
(75 \%)\end{array}$} & \multirow{3}{*}{$\begin{array}{c}\mathrm{p} \\
* \mathrm{NS}\end{array}$} & \multirow{2}{*}{$\begin{array}{c}\begin{array}{c}\mathrm{PI} \\
<=50 \%\end{array} \\
1 \\
(83,3 \%)\end{array}$} & \multirow{2}{*}{$\begin{array}{c}\begin{array}{c}\mathrm{PI}> \\
50 \%\end{array} \\
11 \\
(91,7 \%)\end{array}$} & \multirow{3}{*}{$\begin{array}{c}\mathrm{p} \\
\text { *NS }\end{array}$} & \multirow{2}{*}{$\begin{array}{c}\begin{array}{c}\mathrm{PI} \\
<=50 \%\end{array} \\
4 \\
(33,3 \%)\end{array}$} & \multirow{2}{*}{$\begin{array}{c}\mathrm{PI}> \\
50 \% \\
8 \\
(66,7 \%) \\
\end{array}$} & \multirow{2}{*}{$\begin{array}{c}\mathrm{p} \\
{ }^{*} \mathrm{NS}\end{array}$} \\
\hline Age & $<=60$ & & & & & & & & & \\
\hline & $>60$ & $\begin{array}{c}7 \\
(29,2 \%\end{array}$ & $\begin{array}{c}17 \\
(70,8 \%)\end{array}$ & & $\begin{array}{c}4 \\
(16,7 \%)\end{array}$ & $\begin{array}{c}20 \\
(83,3 \%)\end{array}$ & & $\begin{array}{c}10 \\
(41,7 \%)\end{array}$ & $\begin{array}{c}14 \\
(58,3 \%)\end{array}$ & \\
\hline \multirow[t]{2}{*}{ Sex } & Male & $7(28 \%)$ & $\begin{array}{c}18 \\
(72 \%)\end{array}$ & \multirow[t]{2}{*}{ *NS } & $\begin{array}{c}3 \\
(12 \%)\end{array}$ & $\begin{array}{c}22 \\
(88 \%)\end{array}$ & \multirow[t]{2}{*}{ *NS } & $\begin{array}{c}7 \\
(28 \%)\end{array}$ & $18(72 \%)$ & \multirow[t]{2}{*}{ *NS } \\
\hline & Female & $\begin{array}{c}3 \\
(27,3 \%)\end{array}$ & $\begin{array}{c}8 \\
(72,7 \%)\end{array}$ & & $\begin{array}{c}2 \\
(18,2 \%)\end{array}$ & $\begin{array}{c}9 \\
(81,8 \%)\end{array}$ & & $\begin{array}{c}7 \\
(63,6 \%)\end{array}$ & $\begin{array}{c}4 \\
(36,4 \%)\end{array}$ & \\
\hline \multirow[t]{3}{*}{$\begin{array}{l}\text { lumor } \\
\text { localization }\end{array}$} & $\begin{array}{c}1 / 3 \text { of up } \\
\text { part }\end{array}$ & $\begin{array}{c}1 \\
(33,3 \%)\end{array}$ & $\begin{array}{c}2 \\
(66,7 \%)\end{array}$ & \multirow[t]{3}{*}{ *NS } & $\begin{array}{c}0 \\
(0 \%)\end{array}$ & $\begin{array}{c}3 \\
(100 \%)\end{array}$ & \multirow[t]{3}{*}{ *NS } & $\begin{array}{c}1 \\
(33,3 \%)\end{array}$ & $\begin{array}{c}2 \\
(66,7 \%)\end{array}$ & \multirow[t]{3}{*}{ *NS } \\
\hline & $\begin{array}{c}1 / 3 \text { of } \\
\text { middle part }\end{array}$ & $\begin{array}{c}4 \\
(25 \%)\end{array}$ & $\begin{array}{c}12 \\
(75 \%)\end{array}$ & & $\begin{array}{c}4 \\
(25 \%)\end{array}$ & $\begin{array}{c}12 \\
(75 \%)\end{array}$ & & $\begin{array}{c}8 \\
(50 \%)\end{array}$ & $\begin{array}{c}8 \\
(50 \%)\end{array}$ & \\
\hline & $\begin{array}{c}1 / 3 \text { of } \\
\text { middle part }\end{array}$ & $\begin{array}{c}5 \\
(29,4 \%)\end{array}$ & $\begin{array}{c}12 \\
(70,6 \%)\end{array}$ & & $\begin{array}{c}1 \\
(5,9 \%) \\
\end{array}$ & $\begin{array}{c}16 \\
(94,1 \%)\end{array}$ & & $\begin{array}{c}5 \\
(29,4 \%)\end{array}$ & $\begin{array}{c}12 \\
(70,6 \%)\end{array}$ & \\
\hline \multirow[t]{3}{*}{$\begin{array}{l}\text { Depth of } \\
\text { invasion }\end{array}$} & pll & $\begin{array}{c}3 \\
(100 \%)\end{array}$ & $\begin{array}{c}0 \\
(100 \%)\end{array}$ & \multirow[t]{3}{*}{0.009} & $\begin{array}{c}3 \\
(100 \%)\end{array}$ & $\begin{array}{c}0 \\
(0 \%)\end{array}$ & \multirow[t]{3}{*}{0.00001} & $\begin{array}{c}3 \\
(100 \%)\end{array}$ & $\begin{array}{c}0 \\
(0 \%)\end{array}$ & \multirow[t]{3}{*}{0.06} \\
\hline & pT2 & $\begin{array}{c}1 \\
(50 \%)\end{array}$ & $\begin{array}{c}1 \\
(50 \%)\end{array}$ & & $\begin{array}{c}1 \\
(50 \%)\end{array}$ & $\begin{array}{c}1 \\
(50 \%)\end{array}$ & & $\begin{array}{c}1 \\
(50 \%)\end{array}$ & $\begin{array}{c}8 \\
(50 \%)\end{array}$ & \\
\hline & $\mathrm{pT} 3 / 4$ & $\begin{array}{c}6 \\
(19,4 \%) \\
\end{array}$ & $\begin{array}{c}25 \\
(80,6 \%) \\
\end{array}$ & & $\begin{array}{c}1 \\
(3,2 \%)\end{array}$ & $\begin{array}{c}30 \\
(96,7 \%) \\
\end{array}$ & & $\begin{array}{c}10 \\
(32,3 \%) \\
\end{array}$ & $\begin{array}{c}21 \\
(67,7 \%) \\
\end{array}$ & \\
\hline \multirow[t]{2}{*}{$\mathrm{pN}$} & $\mathrm{Nl}$ & $\begin{array}{c}7 \\
(38,9 \%)\end{array}$ & $\begin{array}{c}11 \\
(91,1 \%)\end{array}$ & \multirow[t]{2}{*}{0.0005} & $\begin{array}{c}5 \\
(27,8 \%)\end{array}$ & $\begin{array}{c}13 \\
(72,2 \%)\end{array}$ & \multirow[t]{2}{*}{0.000} & $\begin{array}{c}10 \\
(55,6 \%)\end{array}$ & $\begin{array}{c}8 \\
(44,4 \%)\end{array}$ & \multirow[t]{2}{*}{0.007} \\
\hline & N2 & $\begin{array}{c}3 \\
(16,7 \%)\end{array}$ & $\begin{array}{c}15 \\
(83,3 \%)\end{array}$ & & $\begin{array}{c}0 \\
(0 \%)\end{array}$ & $\begin{array}{c}18 \\
(100 \%)\end{array}$ & & $\begin{array}{c}4 \\
(22,2 \%)\end{array}$ & $\begin{array}{c}14 \\
(77,8 \%)\end{array}$ & \\
\hline \multirow[t]{4}{*}{$\begin{array}{l}\text { Bormann's } \\
\text { classification }\end{array}$} & I & $\begin{array}{c}0 \\
(0 \%) \\
\end{array}$ & $\begin{array}{c}0 \\
(0 \%) \\
\end{array}$ & \multirow[t]{4}{*}{ *NS } & $\begin{array}{c}0 \\
(0 \%) \\
\end{array}$ & $\begin{array}{c}0 \\
(0 \%) \\
\end{array}$ & $* \mathrm{NS}$ & $\begin{array}{c}0 \\
(0 \%) \\
\end{array}$ & $\begin{array}{c}0 \\
(0 \%) \\
\end{array}$ & $* \mathrm{NS}$ \\
\hline & II & $\begin{array}{c}1 \\
(16,7 \%)\end{array}$ & $\begin{array}{c}5 \\
(83,3 \%) \\
\end{array}$ & & $\begin{array}{c}1 \\
(16,7 \%)\end{array}$ & $\begin{array}{c}5 \\
(83,3 \%) \\
\end{array}$ & & $\begin{array}{c}3 \\
(50 \%) \\
\end{array}$ & $\begin{array}{c}3 \\
(50 \%)\end{array}$ & \\
\hline & III & $\begin{array}{c}6 \\
(30 \%)\end{array}$ & $\begin{array}{c}14 \\
(70 \%)\end{array}$ & & $\begin{array}{c}4 \\
(20 \%)\end{array}$ & $\begin{array}{c}16 \\
(80 \%)\end{array}$ & & $\begin{array}{c}7 \\
(35 \%)\end{array}$ & $\begin{array}{c}13 \\
(65 \%)\end{array}$ & \\
\hline & IV & $\begin{array}{c}3 \\
(30 \%)\end{array}$ & $\begin{array}{c}7 \\
(70 \%)\end{array}$ & & $\begin{array}{c}0 \\
(0 \%)\end{array}$ & $\begin{array}{c}10 \\
(100 \%)\end{array}$ & & $\begin{array}{c}4 \\
(40 \%)\end{array}$ & $\begin{array}{c}6 \\
(60 \%)\end{array}$ & \\
\hline $\begin{array}{l}\text { Lauren's } \\
\text { classification }\end{array}$ & $\begin{array}{l}\text { Intestinal } \\
\text { type }\end{array}$ & $\begin{array}{c}8 \\
(32 \%) \\
\end{array}$ & $\begin{array}{c}17 \\
(68 \%) \\
\end{array}$ & *NS & $\begin{array}{c}5 \\
(20 \%)\end{array}$ & $\begin{array}{c}20 \\
(80 \%)\end{array}$ & *NS & $\begin{array}{c}10 \\
(40 \%)\end{array}$ & $\begin{array}{c}15 \\
(60 \%)\end{array}$ & *NS \\
\hline & Diffuse type & $\begin{array}{c}2 \\
(18,2 \%)\end{array}$ & $\begin{array}{c}9 \\
(81,8 \%)\end{array}$ & & $\begin{array}{c}0 \\
(0 \%)\end{array}$ & $\begin{array}{c}11 \\
(100 \%)\end{array}$ & & $\begin{array}{c}4 \\
(36,4 \%)\end{array}$ & $\begin{array}{c}7 \\
(63,6 \%)\end{array}$ & \\
\hline $\begin{array}{l}\text { Goseki's } \\
\text { classification }\end{array}$ & I & $\begin{array}{c}2 \\
(66,7 \%) \\
\end{array}$ & $\begin{array}{c}1 \\
(33,3 \%) \\
\end{array}$ & *NS & $\begin{array}{c}1 \\
(33,3 \%)\end{array}$ & $\begin{array}{c}2 \\
(66,7 \%) \\
\end{array}$ & "NS & $\begin{array}{c}1 \\
(33,3 \%)\end{array}$ & $\begin{array}{c}2 \\
(66,7 \%)\end{array}$ & $* \mathrm{NS}$ \\
\hline & II & $\begin{array}{c}3 \\
(33,3 \%) \\
\end{array}$ & $\begin{array}{c}6 \\
(66,7 \%) \\
\end{array}$ & & $\begin{array}{c}2 \\
(22,2 \%)\end{array}$ & $\begin{array}{c}7 \\
(77,8 \%) \\
\end{array}$ & & $\begin{array}{c}3 \\
(33,3 \%) \\
\end{array}$ & $\begin{array}{c}6 \\
(66,7 \%) \\
\end{array}$ & \\
\hline & III & $\begin{array}{c}2 \\
(25 \%) \\
\end{array}$ & $\begin{array}{c}6 \\
(75 \%) \\
\end{array}$ & & $\begin{array}{c}1 \\
(12,5 \%)\end{array}$ & $\begin{array}{c}7 \\
(87,5 \%)\end{array}$ & & $\begin{array}{c}3 \\
(37,5 \%)\end{array}$ & $\begin{array}{c}5 \\
(62,5 \%)\end{array}$ & \\
\hline & IV & $\begin{array}{c}3 \\
(18,8 \%)\end{array}$ & $\begin{array}{c}13 \\
(81,2 \%)\end{array}$ & & $\begin{array}{c}1 \\
(6,3 \%)\end{array}$ & $\begin{array}{c}15 \\
(93,7 \%)\end{array}$ & & $\begin{array}{c}7 \\
(43,8 \%)\end{array}$ & $\begin{array}{c}9 \\
(56,2 \%)\end{array}$ & \\
\hline $\begin{array}{l}\text { Histological } \\
\text { differentiation }\end{array}$ & $\mathrm{G} 2$ & $\begin{array}{c}3 \\
(25 \%) \\
\end{array}$ & $\begin{array}{c}9 \\
(75 \%) \\
\end{array}$ & *NS & $\begin{array}{c}2 \\
(16,7 \%)\end{array}$ & $\begin{array}{c}10 \\
(83,3 \%)\end{array}$ & *NS & $\begin{array}{c}3 \\
(25 \%) \\
\end{array}$ & $\begin{array}{c}9 \\
(75 \%) \\
\end{array}$ & *NS \\
\hline & G3 & $\begin{array}{c}7 \\
(29,2 \%)\end{array}$ & $\begin{array}{c}17 \\
(70,8 \%)\end{array}$ & & $\begin{array}{c}3 \\
(12,5 \%)\end{array}$ & $\begin{array}{c}21 \\
(87,5 \%)\end{array}$ & & $\begin{array}{c}11 \\
(45,8 \%)\end{array}$ & $\begin{array}{c}13 \\
(54,2 \%)\end{array}$ & \\
\hline
\end{tabular}

*NS - non statistical significence 
Statistical analysis of correlations between survival time of patients and Ki-67 PI showed a considerably prolonged survival time in the group of patients with $\mathrm{Ki}-67 \mathrm{PI} \leq 50 \%$ in the main mass of tumour and in metastatic lymph node (84 months), as compared to the patients with Ki-67 PI $>50 \%$ (61 months). However, the correlation was not statistically significant $(p=0.81 ; p=0.88$, respectively) (Fig. 1). The correlation between survival time and PCNA PI in gastric cancer was on borderline of statistical significance $(\mathrm{p}=0.06)$. Survival time of patients with PCNA PI in the main mass of tumour $\leq 50 \%$ was by 23 months longer as compared to those with PCNA PI $>50 \%$ (Fig. 2). There was no statistically significant correlation between survival time and PCNA index in metastatic lymph node $(p=0.52)$. Distinct differences, though not statistically significant, were observed in the survival time between patients with MCM2 index $\leq 50 \%$ (84 months) and $>50 \%$ (61 months) in the main mass of tumour ( $\mathrm{p}=0.80)$ (Fig. 3). In patients with metastatic lymph node invasion, the mean survival time did not differ between those with MCM2 $\leq 50 \%$ and $>50 \%$ (approximately 60 months; $\mathrm{p}=0.54$ ).

\section{Discussion}

Ki-67 and PCNA have been found to be the most reliable markers of cell proliferation. MCM2, a novel marker, seems to be more sensitive as compared to other markers long used in routine diagnostics. It is possible to examine the fraction of growth using a routine staining technique in order to assess the correlation between cell proliferation and certain pathomorphological factors in a large retrospective material. Similarly to other authors [12-14], we found no correlation between Ki-67, PCNA and MCM2 PI in the main mass of tumour and certain clinicopathological parameters (Tables 1, 2). On the other hand, Ki-67 PI has been reported to be significantly higher in the intestinal-type cancers by Lauren's classification as compared to the diffuse type $[15,16]$. High PCNA PI has been more frequent in the intestinal than in the diffuse type [17]. Moreover, some authors have shown a significantly higher proliferation index of Ki-67 in high-grade tumours as compared to the low-grade ones [4]. Other clinicopathological factors, including lymph node involvement and depth of invasion have not been found to correlate with Ki-67 index [18,19]. Like other researchers [2,19-23], we found a statistically significant correlation of Ki-67, PCNA and MCM2 PI in advanced gastric cancer with local lymph node involvement. The mean proliferation indices for Ki-67 and PCNA were higher in gastric cancer with local lymph node involvement. Thus, the determination of proliferative activity by means of cell proliferation markers in gastric carcinomas seems to be useful for

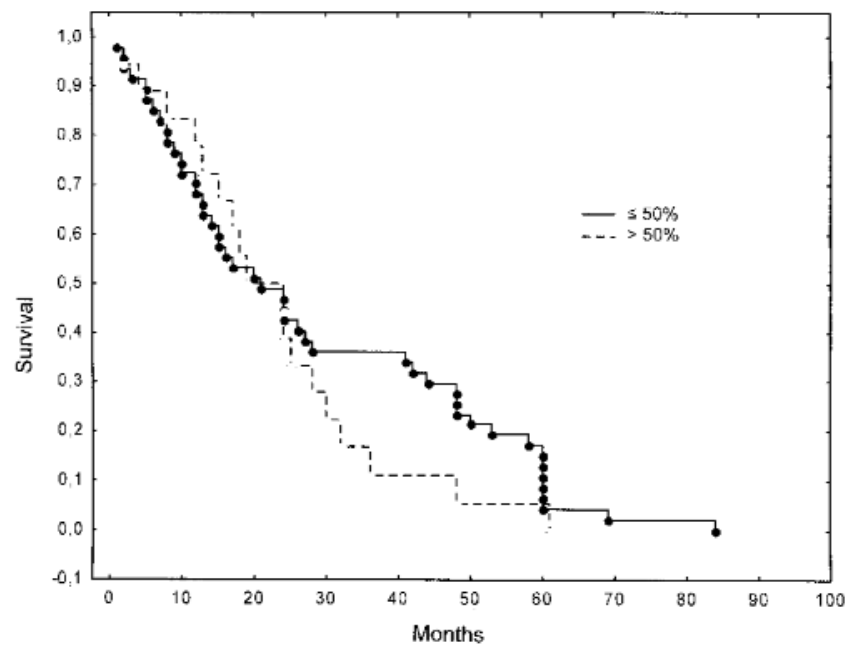

Fig. 1. Correlation of Ki-67 PI in main mass of tumor with survival of patients with advanced gastric cancer.

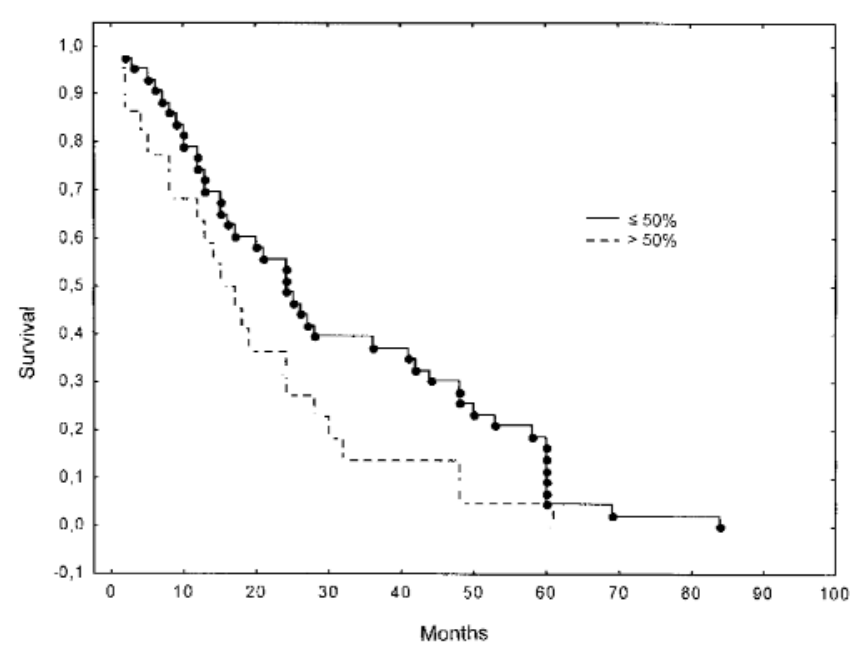

Fig. 2. Correlation of PCNA PI in main mass of tumor with survival of patients with advanced gastric cancer.

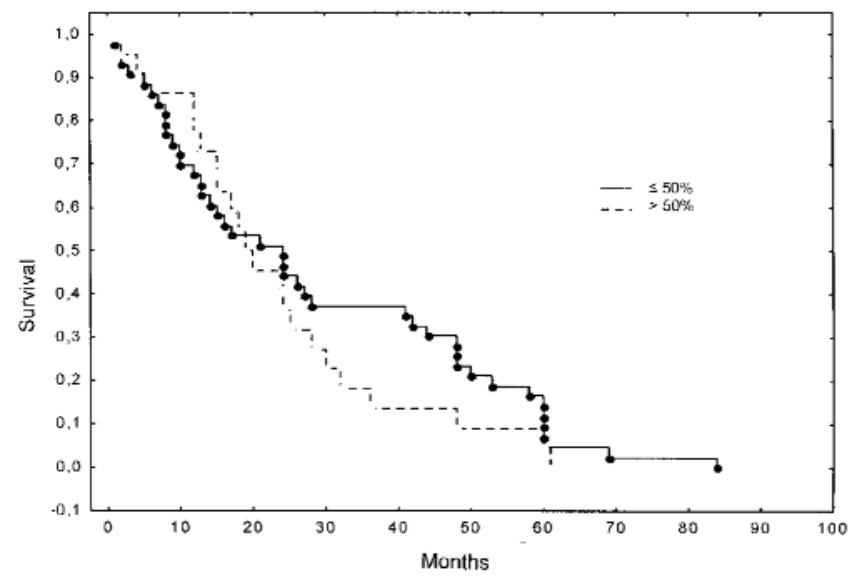

Fig. 3. Correlation of MCM2 PI in main mass of tumor with survival of patients with advanced gastric cancer. 


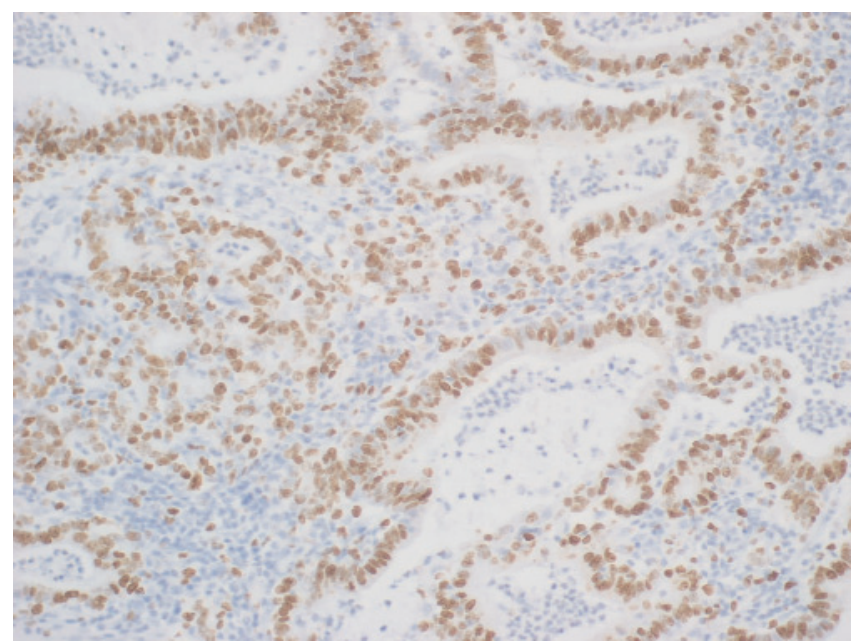

Fig. 4. Positive immunostaining of Ki-67 in primary tumor.

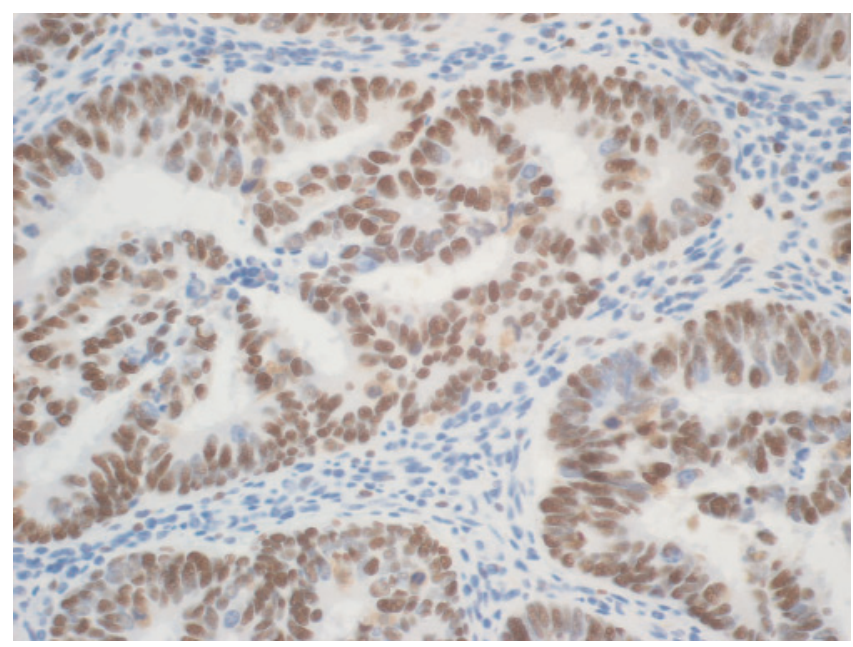

Fig. 5. Positive immunostaining of PCNA in primary tumor.

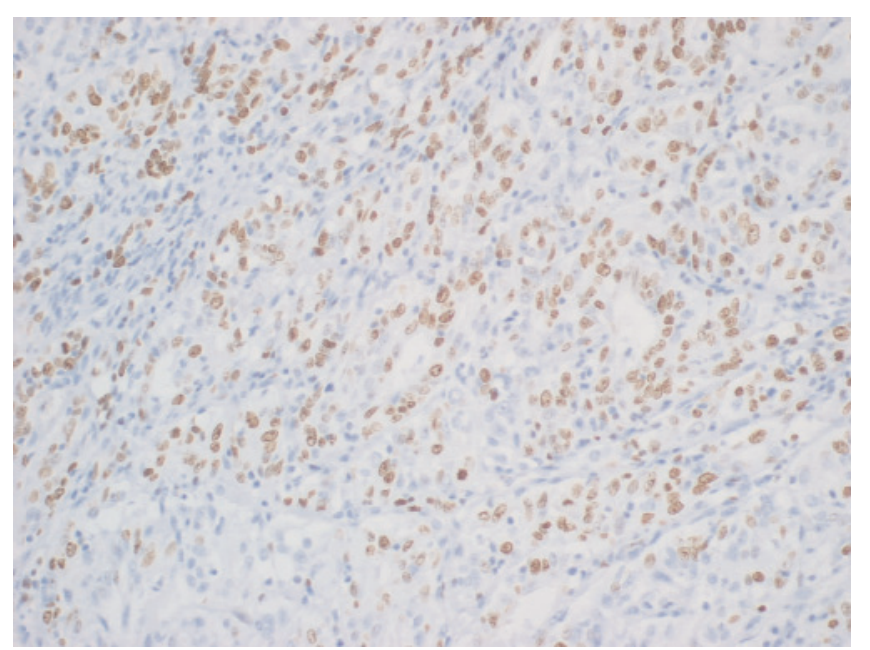

Fig. 6. Positive immunostaining of MCM2 in primary tumor. the prediction of local lymph node involvement $[2,21,22]$. Mita et al. [24] revealed a strong correlation between the proliferative activity of tumour cells and the presence of metastases to lymph nodes and prognosis in cancers. Moreover, these authors demonstrated that tumours with high proliferative activity had high lymph node metastatic potential [24]. Some authors found no correlation between the proliferation index of cell proliferation markers and local lymph node involvement $[15,18]$. In our study, we revealed a statistically significant relationship between histological grading and PCNA index in the main mass of tumour. Irrespective of the grade, the PCNA index was $\leq 50 \%$ in $60 \%$ of the study cases and $>50 \%$ in $40 \%$. PCNA PI was low mainly in higher-grade tumours (G2). Our data are consistent with those published by Aoyagi et al. [25], who observed a significant correlation of PCNA PI with depth of invasion, histological differentiation and the clinical stadium of the disease, thus stating that PCNA index is a good prognostic factor in gastric cancers. Many investigators have found Ki-67 and PCNA to be prognostic factors in tumours of the stomach [13-15,22,23,26-29]. Wong et al. [30] showed Ki-67 PI to be an independent unfavourable prognostic factor in patients with low-differentiated tumours [13]. Igarashi et al. [31] reported a significantly higher value of Ki-67 PI in patients with advanced gastric carcinomas as compared to those with early tumours. The latter group had a considerably longer survival time than the advanced cancer patients. The proliferative activity of cancer cells was found to correlate with metastasis formation and prognosis. It has been reported that $\mathrm{Ki}-67$ can be used as a marker of proliferative activity in gastric cancer and potential metastases to distant organs [21,22]. High PCNA PI has been usually considered to be a reliable marker of tumour progression [14]. Patients with high PCNA PI in early gastric carcinoma much more frequently have lymph node metastases. Moreover, the postoperative survival time of patients with high PCNA PI in gastric carcinoma is considerably shorter than in the case of low PCNA PI in tumour tissue $[23,25]$. Müller et al. [15] observed no difference in the length of postoperative survival between patients with low and high Ki-67 index. Igarashi et al. [31] demonstrated that Ki-67 and PCNA PI correlate with the number of mitoses but are not a reliable prognostic factor in gastric carcinoma. Lee et al. [17] did not find any prognostic significance of PCNA in this cancer. In our study, no correlation was noted between Ki-67 PI and survival time of advanced gastric carcinoma patients. The findings indicate a relationship of Ki-67, PCNA and MCM2 proliferation indices with such malignancy and progression parameters as depth of wall invasion and lymph node involvement. Moreover, a correlation was found between PCNA PI and 
histological grade. Worthy of note is that low proliferative activity, and thus low PCNA PI, indicates more favourable prognosis and longer survival time in patients after tumour resection. Literature data concerning MCM2 PI in the alimentary tract carcinomas suggest that this marker is more sensitive and better for the assessment of the growth fraction in comparison with Ki-67 or PCNA [32,33]. Furthermore, high MCM2 PI in the alimentary tract carcinomas has been found to indicate more unfavourable prognosis and higher biological aggressiveness of tumour [32,34]. The postoperative survival time of patients with high MCM2 PI was significantly shorter as compared to those with its low index [32]. The data seem to be consistent with the findings obtained for prostate carcinoma [35], non-small-cell carcinoma of the lung [36] and carcinoma of the kidney [37]. We could not find any literature data concerning correlations between MCM2 PI in gastric carcinomas and pathomorphological parameters. Our results seem to indicate a correlation of MCM2 PI in advanced gastric carcinomas with such parameters of histological malignancy as depth of invasion and local lymph node involvement. Patients with low MCM2 PI in the main mass of tumour were found to live longer as compared to those with high PI. Thorough investigation into the relationship between MCM2 PI and respective clinicopathological parameters may allow application of this protein in routine diagnostics. Assessment of correlations of MCM2 index with other markers, such as Ki-67 or PCNA, may help determine whether the MCM2 protein is a more sensitive and reliable marker in the case of advanced gastric carcinomas. Our results seem to indicate the relationship of Ki-67, PCNA and MCM2 PI with such parameters of histological malignancy as depth of wall invasion and lymph node metastases. However, correlation between the proliferation index and postoperative survival time was observed only for PCNA.

\section{References}

[ 1] Kelley JR, Duggon JM. Gastric cancer epidemiology and risk factors. Commentary. J Clin Epidemiology. 2003;56:1-9.

[2] Kabashima A, Maehara Y, Koga T, Kakeji Y, Sugimachi K. The biologic features of intramucosal gastric carcinoma with lymp node metastasis. Surgery. 2002;131:S71-S77.

[3] Gerdes J, Lemke H, Baisch H, Wacker HH, Schwab U, Stein $\mathrm{H}$. Cell cycle analysis of the cell proliferation- associated human nuclear antigen defined by the monoclonal antibody Ki-67. J Immunol. 1984;133:1710-1715.

[4] Niimi Ch, Goto H, Ohmiya N, Niwa Y, Hayakawa T, Nagasaka T, Nakashima N. Usefulness of p53 and Ki-67 immunohistochemical analysis for preoperative diagnosis of extremaly well- differentiated gastric adenocarcinoma. Am J Clin Pathol. 2002;118:683-692.

[ 5] Ino H, Chiba T. Expression of proliferating cell nuclear antigen (PCNA) in the adult and developing mouse nervous system. Mol Brain Res. 2000;78:163-174.
[6] Takasaki Y, Fishwild D, Tan EM. Characterisation of proliferating cell nuclear antigen recognized by autoantibodies in lupus sera. J Exp Med. 1984;159:981-992.

[ 7] Skotnicka- Klonowicz G,Kobos J, Trejster E, Szymik- Kantorowicz S, Daszkiewicz P. Prognostic value of proliferating cell nuclear antygen in Wilm's tumour in children. EJSO. 2002;28:67-71.

[ 8] Kodoni I, Osaki M, Shomori K, Araki K, Goto E, Ryoke K, Ito $\mathrm{H}$. Minichromosome maintenance 2 expression is correlated with mode of invasion and prognosis in oral squamous cell carcinoma. J Oral Pathol Med. 2002;32:468-474.

[ 9] Bailis JM, Forsburg SL. MCM proteins: DNA damage, mutagenesis and repair. Current Opinion in Genetics \&Development. 2004;14:17-21.

[10] Wuarin J, Nurse P. Regulating S phase: CDKs, licensing and proteolysis. Cell. 1996;85:785-787.

[11] Tan DF, Huberman JA, Hyland A, Loewen GM, Brooks JS, Beck AF, Todorov IT, Bepler G. MCM2- a promising marker for premalignant lesions of the lung: a cohort study. $B M C$ Cancer. 2001;1:6-12.

[12] Oshima CT, Iriya K, Forones NM. Ki-67 as a prognostic marker in colorectal cancer but not in gastric cancer. Neoplasma. 2005;52:420-424.

[13] Kikuyama S, Kubota T, Shimizu K, Miyazakita M. Ki-67 antigen expression in relation to clinicopathological variables and prognosis in gastric cancer. Oncol Rep. 1998;5:867-870.

[14] Wu K, Zhao L, Li Y, Shan Y-J, Wu L-J. Effects of vitamin E succinate on the expression of FAS and PCNA proteins in human gastric carcinoma cells and its clinical signifecence. J Gastroenterol. 2004;10:945-949.

[15] Müller W, Schneiders A, Meier S, Hommel G, Gabbert HE. Immunohistochemical study on the prognostic value of MIB1 in gastric carcinoma. British J Cancer. 1996;74:759-765.

[16] Jian S, Filipe MI, Hall PA, Waseem N, Lane DP, Levison DA. Prognostic value of proliferating cell nuclear antigen in gastric carcinoma. J Clin Pathol. 1991;44:655-659.

[17] Lee KE, Lee H-J, Kim YH, Yu HJ, Yang H-K, Kim W-H, Lee $\mathrm{KU}$, Choe KJ, Kim J-P. Prognostic signifeicence of p53, nm23, PCNA and c-erbB-2 in gastric cancer. Jap J Clin Oncol. 2003;33:173-179.

[18] Xu L, Zhang S-M, Wang Y-P, Zhao F-K, Wu D-Y, Xin. Relation between DNA ploidy, expression of Ki-67 antigen and gastric cancer metastasis. W J Gastroenterol. 1999;5: 10-11.

[19] Elpek GO, Gelen T, Aksoy NH, Karpuzoglu T, Keleş N. Microvessel count, proliferating cell nuclear antign and Ki-67 indices in gastric adenocarcinoma. Pathol Oncol Res. 2000;6: 59-64.

[20] Terada R, Yasutake T, Nakamura S, Hisamatsu T, Nakagoe T, Ayabe H, Tagawa Y. Evaluation of metastatic potential of gastric tumors by staining for proliferating cell nuclear antigen and chromosome 17 numeral aberrations. Ann Surg Oncol. 2000;8:525-532.

[21] Inadomi T, Tan M, Suzuki H, Shigematsu C. Immunohistochemical evaluation of the probability if skin metastasis in gastric cancer. Eur J Dermatol. 1999;9:214-217.

[22] Lei XU, Su-Min Z, Yan-Ping W, Feng-Kai Z, Dong-Ying WU, Yan XIN. Relationship between DNA ploidy, expression of Ki-67 antigen and gastric cancer metastasis. World J Gastroenterol. 1999;5:10-11.

[23] Noda H, Maehara Y, Irie K, Kakeji Y, Yonemura T, Sugimachi $\mathrm{K}$. Increased proliferative activity caused by loss p21 (WAF1/CIP1) expression and its clinical significance in patients with early-stage gastric carcinoma. Cancer. 2002;94: 2107-2112.

[24] Mita T, Shimoda T. Risk factors for lymph node metastasis of submucosal invasive differentiated type gastric carcinoma: cilinical significance of histological heterogeneity. $J$ Gastroenterol. 2001;36:661-668. 
[25] Aoyagi K, Kohfuji K, Yano S, Murakami N, Miyagi M, Takeda J, Shirouzu K. The expression of proliferating cell nuclear antigen, p53, p21 and apoptosis in primary gastric lymphoma. Surgery. 2002;132:20-26

[26] Toquet C, Le Neel JC, Guillou L, Renaudin K, Hamy A, Heymann M-F, Simon-Valla S, Le Borgne J, Maugard C, Fiche M. Elevated (?10\%) MIB-1 proliferative indexcorrelates with poor outcome in gastric stromal tumor patients. Dig Dis Sci. 2002:47:2247-2253.

[27] Belessi C, Parasi AS, Manioudaki HS, Laoutaris NP, Legakis NC, Peros GT, Androulakis GA. Prognostic impact of DNA ploidy pattern, S-phase fraction (SPF) and proliferating Cell nuclear antigen (PCNA) in patients with primary gastric lymphoma. J Surg Oncol. 2003;82:247-255.

[28] Jiang Y-A, Zhang Y-Y, Luo H-S, Xing S-F. Mast cell density and the context of clinicopathological parameters and expression of $\mathrm{p} 185$, estrogen receptor and proliferating cell nuclear antigen in gastric carcinoma. World J Gastroenterol. 2002;8: 1005-1008.

[29] Konno S, Takebayashi Y, Aiba M, Akiyama S, Ogawa K. Clinicopathological and prognostic significence of thymidine phosphorylatse and proliferating cell nuclear antygen in gastric carcinoma. Cancer Lett. 2001;166:103-111.

[30] Wong NA, Young R, Malcomson RD, Nayar AG, Jamieson LA, Save VE, Carey FA, Brewster DH, Han C, Al-Nafussi A. Prognostic indicators for gastrointestinal stromal tumors: a clinicopathological and immunohistochemical study of 108 resected cases of the stomach. Histopathology. 2003;43:118126.

[31] Igarashi N, Takahashi M, Ohkubo H, Omata K, Iida R, Fujimoto S. Predictive value of Ki-67, p53 protein and DNA content in the diagnosis of gastric carcimona. Cancer. 1999;86: 1449-1454.
[32] Kato H, Miyazaki T, Fukai Y, Nakajima M, Sohda M, Takita J, Maruda N, Fukuchi M, Manda R, Ojima H, Tsukada K, Asao T, Kuwano H. A new proliferation marker, minichromosome maintenance protein 2 , is associated with tumor aggressiveness in esophageal squamous cell carcinoma. J Surg Oncol. 2003;84:24-30.

[33] Chatrath P, Scott IS, Morris LS, Davies RJ, Rushbrook SM, Bird K, Fowler DI, Grant JW, Saeed IT, Howard D, Laskey RA, Coleman N. Aberrent expression of minichromosome maintenance protein- 2 and $\mathrm{Ki}-67$ in laryngel squamous epithelial lesions. Br J Cancer. 2003; 89: 1048-1054.

[34] Scott IS, Morris LS, Bird K, Davies RJ, Volwer SL, Rushbrook SM, Marshall AE, Laskey RA, Miller R, Arends MJ, Coleman N. A novel immunohistochemical method to estimate cell-cycle phase distriburion in archival tissue: implicatons for the prediction of outcome in colorectal cancer. J Parhol. 2003;210:187-197.

[35] Meng MV, Grossfeld GD, Williams GH, Dilworth S, Stoeber K, Mulley TW, Weinberg V, Carroll PR, Tlsty TD. Minichromosome maintenance protein 2 expression in prostate: characterization ans association with outcome after therapy for cancer. Clin Cancer Res. 2001;7:2712-2718.

[36] Ramnath N, Hernandez FJ, Tan DF, Huberman JA, Natarajan N, Beck AF, Hyland A, Todorov IT, Brooks JS, Bepler G. MCM2 is an independent predictor of survival in patients with non-small-cell lung cancer. J Clin Oncol. 2001;19: 4259-66.

[37] Wharton SB, Chan KK, Anderson JR, Stoeber K, Wiliams $\mathrm{GH}$. Replicative Mcm2 protein as a novel proliferation marker in oligodendrogliomas and its relationship to Ki-67 labeling index, histological grade, and prognosis. Neuropathol Appl Neurobiol. 2001;27:305-313.

Submitted: 28 December, 2008 Accepted after reviews: 11 February, 2009 